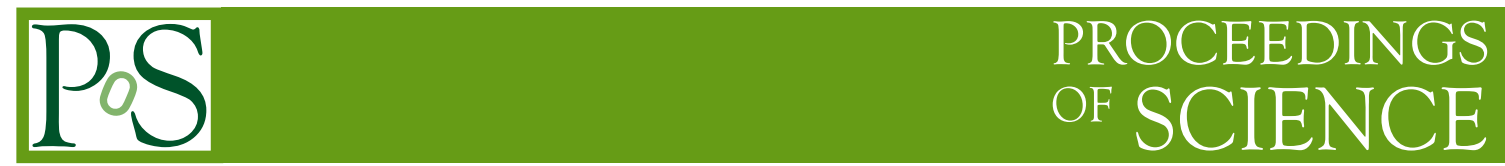

\title{
Systematics in T2HK, T2HKK and DUNE
}

\author{
Osamu Yasuda* \\ Tokyo Metropolitan University \\ E-mail: vasudadphys.se.tmu.ac.70
}

Because of the large statistics, the T2HK, T2HKK and DUNE experiments will be sensitive to systematics. We study the effect of the systematics on the determination of neutrino mass hierarchy, octant of the mixing angle $\theta_{23}$ and $\delta_{C P}$ at these experiments in the standard three flavor scenario and also analyze the role of systematic uncertainties in constraining the parameters of the non-standard interactions in neutrino propagation.

Neutrino Oscillation Workshop (NOW2018)

9 - 16 September, 2018

Rosa Marina (Ostuni, Brindisi, Italy)

${ }^{*}$ Speaker. 


\section{Introduction}

From the neutrino experiments in the last twenty years, all the mixing angles and the mass squared differences have been measured, and the only unknown quantities which can be probed by neutrino oscillation are the mass hierarchy pattern, octant of the mixing angle $\theta_{23}$ and the CP phase $\delta_{C P}$. It is expected that these unknown quantities will be determined in the future neutrino experiments, such as T2HK [四], T2HKK [వ] and DUNE [B]]. It is also expected that these future experiments with intense accelerator neutrino beams will enable us to probe new physics beyond the standard model with massive neutrinos, by looking for the deviation from the standard scenario. Due to the large statistics of the T2HK, T2HKK and DUNE experiments, these experiments will be sensitive to systematics. In this talk I discuss the effect of the systematics in the determination of neutrino mass hierarchy, octant of the mixing angle $\theta_{23}$ and $\delta_{C P}$ in the standard three flavor scenario, as well as the one in constraining the the parameters of the flavor dependent non-standard interactions in neutrino propagation. ${ }^{1}$

The sensitivity of each experiment is estimated with $\chi^{2}$ for Poisson distribution by comparing the true $\left(N^{\text {true }}\right)$ and test $\left(N^{\text {test }}\right)$ events

$$
\chi_{\text {stat }}^{2}=2 \sum_{i}\left[N_{i}^{\text {test }}-N_{i}^{\text {true }}-N_{i}^{\text {true }} \log \left(\frac{N_{i}^{\text {test }}}{N_{i}^{\text {true }}}\right)\right],
$$

where the index $i$ stands for the number of energy bin, and each number $N^{\text {test }}$ of events consists of the signal $S^{\text {test }}$ and the background $B^{\text {test }}: N_{i}^{\text {test }}=S_{i}^{\text {test }}+B_{i}^{\text {test }}$. Here we introduce only the two systematic errors. One is the overall normalization error, and the other one is the tilt error. For simplicity we assume that the systematic errors for the signals and the backgrounds and those for neutrino mode and for antineutrino mode are the same, respectively. ${ }^{2}$ By introducing the pull variables $\xi_{k}(k=1, \cdots, 4), S_{i}^{\text {test }}$ and $B_{i}^{\text {test }}$ are scaled as

$$
S_{i}^{\text {test }} \rightarrow S_{i}^{\text {test }}\left(1+c_{1} \xi_{1}+c_{2} \xi_{2} \frac{E_{i}-E_{\mathrm{av}}}{E_{\max }-E_{\min }}\right), B_{i}^{\text {test }} \rightarrow B_{i}^{\text {test }}\left(1+c_{1} \xi_{3}+c_{2} \xi_{4} \frac{E_{i}-E_{\mathrm{av}}}{E_{\max }-E_{\min }}\right) .
$$

Here the tilt error $c_{2}$ is fixed as 0.1 , while the normalization error $c_{1}$ is varied from 0.01 to 0.07 . The final $\chi^{2}$ is defined by

$$
\chi^{2}=\min _{\xi_{k}}\left(\chi_{\text {stat }}^{2}+\sum_{k=1}^{4} \xi_{k}^{2}\right) .
$$

We analyze the sensitivity both in the case of the standard three flavor scenario and the case with the non-standard interactions. In the latter case, the original time evolution is given by the Hamiltonian

$$
H^{\mathrm{eff}}=\frac{1}{2 E} U \operatorname{diag}\left(0, \Delta m_{21}^{2}, \Delta m_{31}^{2}\right) U^{-1}+\sqrt{2} G_{F} N_{e}\left(\begin{array}{ccc}
1+\varepsilon_{e e} & \varepsilon_{e \mu} & \varepsilon_{e \tau} \\
\varepsilon_{\mu e} & \varepsilon_{\mu \mu} & \varepsilon_{\mu \tau} \\
\varepsilon_{\tau e} & \varepsilon_{\tau \mu} & \varepsilon_{\tau \tau}
\end{array}\right)
$$

\footnotetext{
${ }^{1}$ The details can be found in Ref. [四]. In this talk I discuss T2HKK with the off-axis angle $1.5^{\circ}$ only, whereas other options of T2HKK with $2.5^{\circ}$ and $2.0^{\circ}$ were also considered in Ref. [四].

${ }^{2}$ DUNE collaboration [B] introduces the two kinds of normalization errors $N\left(v_{\mu} \rightarrow v_{\mu}\right) \rightarrow(1+0.05 \alpha) N\left(v_{\mu} \rightarrow v_{\mu}\right)$, $N\left(v_{\mu} \rightarrow v_{e}\right) \rightarrow(1+0.05 \alpha+0.02 \beta) N\left(v_{\mu} \rightarrow v_{e}\right)$, where $\alpha$ and $\beta$ are the pull variables. Here we analyze the sensitivity with only one normalization error for simplicity.
} 
but taking into account of the constraints on $\varepsilon_{\alpha \beta}$, we assume the following ansatz:

$$
\left(\begin{array}{ccc}
1+\varepsilon_{e e} & \varepsilon_{e \mu} & \varepsilon_{e \tau} \\
\varepsilon_{\mu e} & \varepsilon_{\mu \mu} & \varepsilon_{\mu \tau} \\
\varepsilon_{\tau e} & \varepsilon_{\tau \mu} & \varepsilon_{\tau \tau}
\end{array}\right) \rightarrow\left(\begin{array}{cll}
1+\varepsilon_{e e} & 0 & \varepsilon_{e \tau} \\
0 & 0 & 0 \\
\varepsilon_{e \tau}^{*} & 0\left|\varepsilon_{e \tau}\right|^{2} /\left(1+\varepsilon_{e e}\right)
\end{array}\right)
$$

where we have taken into account the constraints on the dimensionless parameters $\varepsilon_{\alpha \beta}$ of the nonstandard interactions, i.e., $\left|\varepsilon_{\mu \alpha}\right| \ll 1[\square],\left|\varepsilon_{e \tau}\right|^{2} \simeq \varepsilon_{\tau \tau}\left(1+\varepsilon_{e e}\right)\left[\right.$ [G]. ${ }^{3}$

\section{Results and Conclusion}

The results in the standard three flavor case are shown in Fig.t (a)-(b) for mass hierarchy, in Fig.】 (c)-(d) for octant, and in Fig.వ (e)-(f) for CP violation. In general T2HK has the largest numbers of events because its baseline length is the smallest, so the dependence of T2HK on the systematic errors is stronger than that of the other two experiments. If the hierarchy is known then we see that the CPV discovery sensitivity of T2HK is better than T2HKK if the systematics is less than $1 \%$.

The results in the case of the non-standard interactions are shown in Fig.D(g)-(h) for sensitivity on $\left(\varepsilon_{e e},\left|\varepsilon_{e \tau}\right|\right)$, and in Fig.】 (i)-(j) for sensitivity on $\left(\delta_{C P}, \arg \left(\varepsilon_{e \tau}\right)\right)$. The sensitivities corresponding to the $1 \%$ and $3 \%$ systematics errors are similar in constraining $\varepsilon_{e e}$ and $\left|\varepsilon_{e \tau}\right|$. In constraining the CP phases, lowering systematic uncertainty below $3 \%$ would not result in a significant improvement in the achieved sensitivity.

Measurement of the systematics plays an important role in the sensitivity reach of the future high statistics long-baseline experiments. While it is expected to be difficult to improve the systematic errors, it is worth making an effort to improve them, since the sensitivity of one experiment could be better than others, depending on the value of the systematics.

\section{Acknowledgments}

This research was partly supported by a Grant-in-Aid for Scientific Research of the Ministry of Education, Science and Culture, under Grants No. 18K03653 and No. 18H05543.

\section{References}

[1] K. Abe et al. [Hyper-Kamiokande Working Group], arXiv:1412.4673 [physics.ins-det].

[2] K. Abe et al. [Hyper-Kamiokande Collaboration], PTEP 2018 (2018) no.6, $063 \mathrm{C} 01$ doi:10.1093/ptep/pty044 [arXiv:1611.06118 [hep-ex]].

[3] R. Acciarri et al. [DUNE Collaboration], arXiv:1512.06148 [physics.ins-det].

[4] M. Ghosh and O. Yasuda, Phys. Rev. D 96 (2017) no.1, 013001 doi:10.1103/PhysRevD.96.013001 [arXiv:1702.06482 [hep-ph]].

[5] C. Biggio, M. Blennow and E. Fernandez-Martinez, JHEP 0908, 090 (2009).

[6] A. Friedland, C. Lunardini and M. Maltoni, Phys. Rev. D 70, 111301 (2004).

\footnotetext{
${ }^{3}$ In Sect. 7 of Ref. [ᄆ]], sensitivity of T2HKK to the non-standard interactions without the ansatz $\varepsilon_{\tau \tau}=\left|\varepsilon_{e \tau}\right|^{2} /(1+$ $\left.\varepsilon_{e e}\right)$ is discussed.
} 

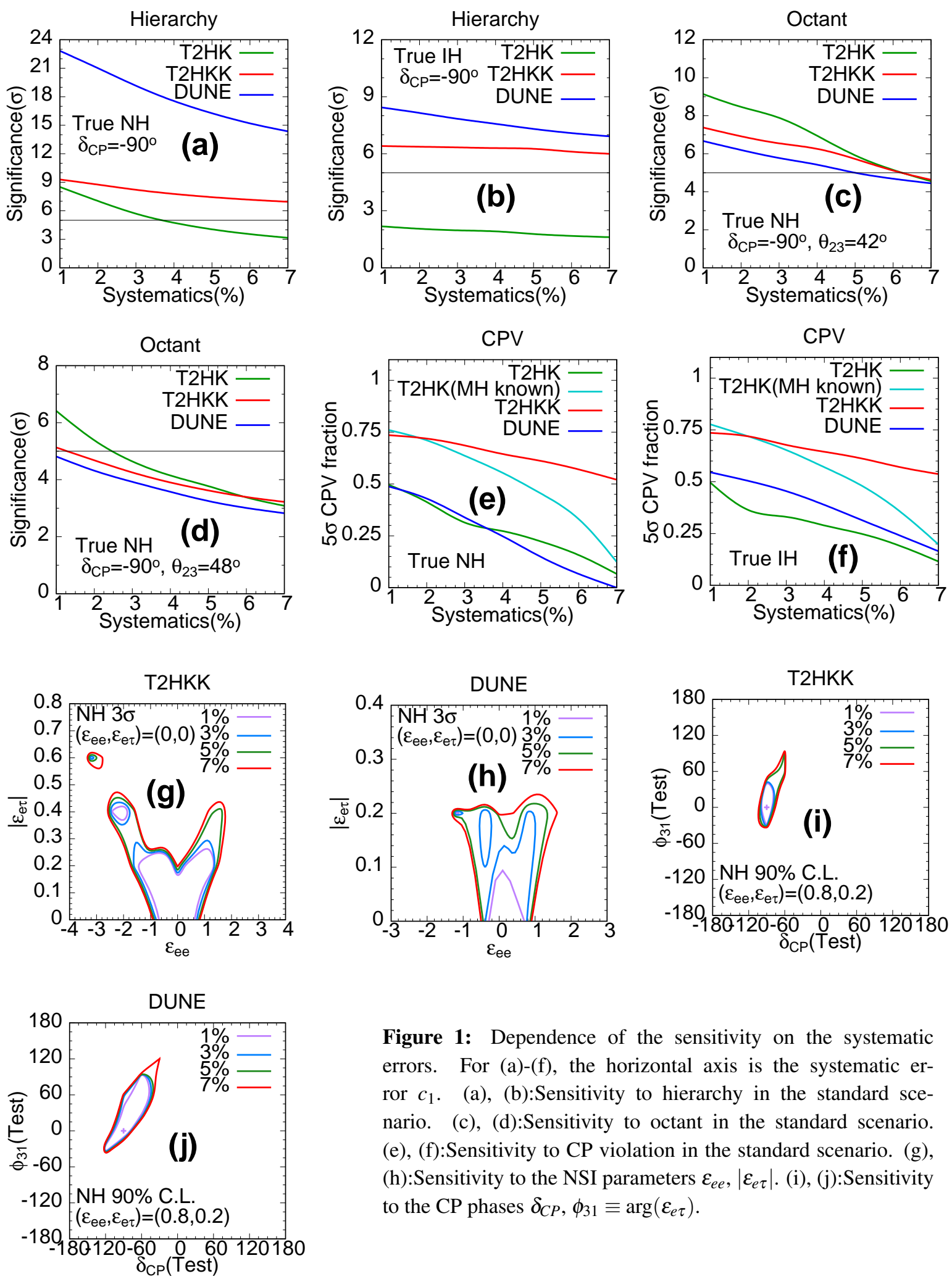

Figure 1: Dependence of the sensitivity on the systematic errors. For (a)-(f), the horizontal axis is the systematic error $c_{1}$. (a), (b):Sensitivity to hierarchy in the standard scenario. (c), (d):Sensitivity to octant in the standard scenario. (e), (f):Sensitivity to CP violation in the standard scenario. (g), (h):Sensitivity to the NSI parameters $\varepsilon_{e e},\left|\varepsilon_{e \tau}\right|$. (i), (j):Sensitivity to the $\mathrm{CP}$ phases $\delta_{C P}, \phi_{31} \equiv \arg \left(\varepsilon_{e \tau}\right)$. 